Article

\title{
Do GNAQ and GNA11 Differentially Affect Inflammation and HLA Expression in Uveal Melanoma?
}

\author{
Christiaan van Weeghel ${ }^{1}$, Annemijn P. A. Wierenga ${ }^{1}$, Mieke Versluis ${ }^{1}\left(\mathbb{D}\right.$, Thorbald van Hall $\left.{ }^{2} \mathbb{(}\right)$, \\ Pieter A. van der Velden ${ }^{1}$, Wilma G. M. Kroes ${ }^{3}$, Ulrich Pfeffer ${ }^{4} \mathbb{D}$, Gregorius P. M. Luyten ${ }^{1}$ and \\ Martine J. Jager $1, * \mathbb{D}$ \\ 1 Department of Ophthalmology, Leiden University Medical Center, Albinusdreef 2, 2333 ZA Leiden, \\ The Netherlands \\ 2 Department of Clinical Oncology, LUMC, 2333 ZA Leiden, The Netherlands \\ 3 Department of Clinical Genetics, LUMC, 2333 ZA Leiden, The Netherlands \\ 4 Tumor Epigenetics, IRCCS Ospedale Policlinico San Martino, 16132 Genova, Italy \\ * Correspondence: m.j.jager@lumc.nl
}

Received: 29 June 2019; Accepted: 31 July 2019; Published: 7 August 2019

\begin{abstract}
Inflammation, characterized by high numbers of infiltrating leukocytes and a high HLA Class I expression, is associated with a bad prognosis in uveal melanoma (UM). We wondered whether mutations in GNA11 or GNAQ differentially affect inflammation and HLA expression, and thereby progression of the disease. We analyzed data of 59 primarily enucleated UM eyes. The type of GNAQ/11 mutation was analyzed using dPCR; chromosome aberrations were determined by Fluorescence in Situ Hybridization (FISH), karyotyping, and single nucleotide polymorphism (SNP) analysis, and mRNA expression by Illumina PCR. Comparing tumors with a GNAQ mutation with those with a GNA11 mutation yielded no significant differences in histopathological characteristics, infiltrate, or HLA expression. When comparing the Q209L mutations with Q209P mutations in tumors with monosomy of chromosome 3, a higher mitotic count was found in the Q209P/M3 tumors $(p=0.007)$. The Kaplan-Meier $(\mathrm{KM})$ curves between the patients of the different groups were not significantly different. We conclude that the type (Q209P/Q209L) or location of the mutation (GNA11/GNAQ) do not have a significant effect on the immunological characteristics of the tumors, such as infiltrate and HLA Class I expression. Chromosome 3 status was the main determinant in explaining the difference in infiltrate and HLA expression.
\end{abstract}

Keywords: uveal melanoma; mutations; chromosome aberrations; mRNA expression; survival

\section{Introduction}

Uveal melanoma (UM) is a form of cancer that occurs mostly in people aged 60 years and older and has a prevalence of around 5.0 per million people within The Netherlands [1]. Unfortunately, between $30 \%$ and $50 \%$ of the people affected by UM eventually die due to metastases [2]. UM forms in the uvea, which is made up of the iris, the ciliary body, and the choroid. At this moment in time, the most common treatments of UM are enucleation, plaque irradiation, and proton therapy.

The primary GNAQ/GNA11 mutation in UM occurs in the melanocyte and is already present in choroidal nevi [3-5]. During malignant progression, this genetic modification is usually followed by gain of chromosome $6 \mathrm{q}$ or gain of $8 \mathrm{q}$, as well as a mutation in the BAP1, EIF1AX, or SF3B1 gene [6-8], and/or a complete loss of one chromosome 3 (monosomy 3 (M3)). M3 loss and mutations in BAP1 often co-occur and are associated with a bad prognosis [9-14]. Chromosome 6q gain is associated with a better prognosis and a lower chance of developing metastatic disease [15]. Gain of 8q is associated 
with metastases but often occurs together with monosomy 3 . The specific sequence of events with regard to BAP1 and chromosome 3 is not yet clear, while it is likely that gain of $8 \mathrm{q}$ often develops prior to loss of chromosome 3 [16-18].

It is thought that UM cells have a survival advantage because of the tumor's location in the immune-privileged eye: tumor cells would not primarily induce a systemic anti-tumor immune response but might even inhibit the development of anti-tumor immunity [19]. However, especially prognostically-bad UM often display an inflammatory phenotype which is characterized by the presence of immune cells such as T lymphocytes, macrophages, and an increased HLA expression [20]. The presence of such an inflammatory phenotype is associated with a bad prognosis [13,21-23].

HLA Class I (A-C and E-G) molecules present tumor proteins to T cells, while HLA Class II molecules (DM, DO, DP, DQ and DR) present proteins that originate from outside the cell to T cells. If the T cells recognize a presented protein that does not belong to the normal set of proteins, an immune reaction can develop. Infiltrating T cells in UM do not prevent the tumor from developing because the infiltrate is seen as a factor associated with a poor prognosis [23,24]. From previous research it is known that HLA-A and HLA-B expression is higher in patients with an M3 status [23]. Natural killer (NK) cells cannot attack the tumor due to the high amount of HLA expressed on UM cells, which inhibits their function, and due to the presence of high levels of macrophage migration inhibitory factor (MIF) and TGF- $\beta$ in the aqueous humor, which inhibit NK cell function [19]. HLA-G can help cancer cells escape the immune system and can also inhibit NK cell-mediated lysis [25]. Due to the lack of response of the immune system to the tumor, the tumor will continue to grow $[19,26]$. However, research from our lab has shown a low expression of HLA-G [27]. From previous research it is known that HLA-A and HLA-B expression is higher in patients with an M3 status [23]. We have previously noticed that an increase in the number of macrophages in the disomy 3 (D3) group is related to the presence of an extra copy of chromosome 8q [28].

M3/BAP1-mutated tumors have distinct genomic signaling and immune profiles [13] and are often associated with high numbers of infiltrating leukocytes and macrophages. However, a great variability in infiltrate has been observed within the M3 and also in the D3 group. We wondered whether the expression of different types of HLA molecules in UM is similarly related to loss of chromosome 3 or might be related to the type of basic mutation (GNAQ or GNA11 mutation or the type of mutation (Q209P/Q209L)). GNAQ and GNA11 are genes encoding subunits of G-protein-coupled receptors (GPCRs), transmembrane receptors that serve as signal transducers operating from the cell membrane. They require glutamine in position 209 (Q209) to function properly. Without glutamine on Q209, the GPCR cannot hydrolyze Guanosine Triphosphate (GTP) to Guanosine Diphosphate (GDP) $[4,29]$. Q209 is a hotspot for missense mutations where the proteins translated from GNAQ/GNA11 often have glutamine replaced by proline (Q209P mutation) or by leucine (Q209L mutation). We set out to determine whether the difference between a GNAQ or GNA11 mutation or the type of mutation in these genes (Q209L versus Q209P) influenced the level of HLA expression and infiltrate in UM.

\section{Results}

\section{1. $m R N A$ and Patient Distribution}

We first compared the expression of different HLA molecules with the tumor's chromosome 3 status using mRNA expression data acquired from an Illumina chip: in Figure 1, it is shown that M3 tumors differ greatly from disomy 3 (D3) tumors with regard to inflammatory markers, with more of the M3 tumors showing high numbers of infiltrating leukocytes and a high HLA Class I expression. However, within each group, tumors differ significantly in infiltrate and HLA expression (Figure 1). For illustration, tumors were placed into a D3 and M3 cluster, and then into clusters through unsupervised clustering, based on the inflammatory and HLA markers within the D3 and M3 groups. 


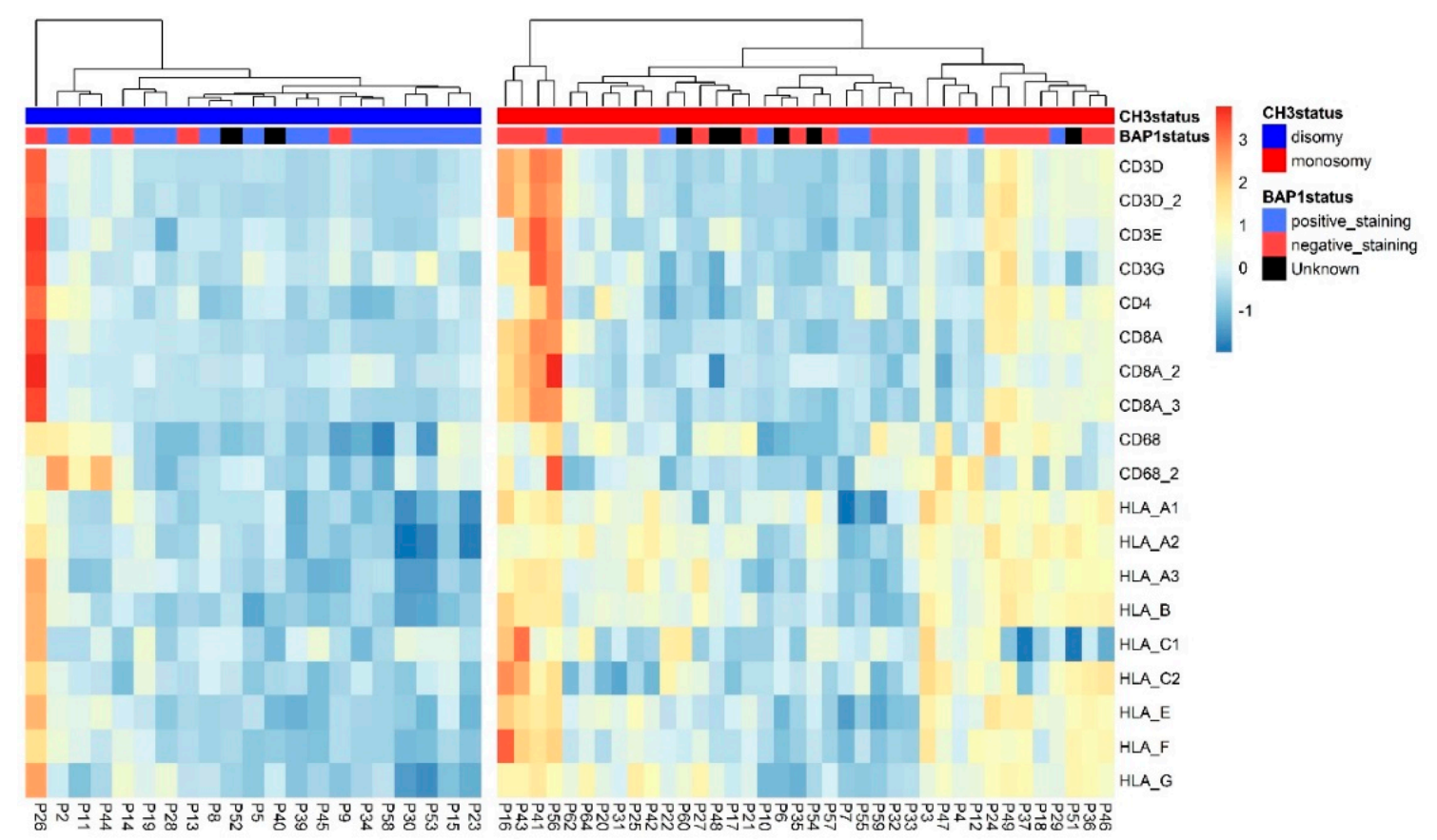

Figure 1. Two heatmaps showing the expression of several infiltrate and HLA Class I markers, normalized per marker over all 59 tumors. On the left is the heatmap of the disomy 3 (D3) tumors ( $n=21)$ and on the right the heatmap of the monosomy 3 (M3) tumors ( $n=38)$; tumors are clustered according to the amount of infiltrate and HLA Class I expression. Annotated are the chromosome 3 status (blue for D3 tumors, red for M3 tumors) and BAP1 staining. The presence of positive nuclear staining for BAP1 is indicated in blue (BAP1 is normal), while red indicates no BAP1 staining and black an unknown BAP1 status.

We set out to determine if mutations in GNAQ and GNA11 are responsible for different degrees of inflammation within the D3 or M3 groups. The comparisons of clinical and histopathological information can be seen in Tables 1 and 2: Table 1 contains the tumors with GNAQ or GNA11 mutations, while Table 2 contains the data of tumors identified by Q209L and Q209P mutations. These data show that the clinical and histopathological characteristics did not differ significantly between mutations.

We subsequently set out to investigate whether the differences in levels of infiltrating leukocytes or in HLA expression were related to the presence of GNAQ/GNA11 mutations (Table 3), or the type of mutation Q209L/Q209P (Table 4). No differences were observed in the expression of inflammatory markers such as HLA expression or levels of infiltrating leukocyte. However, significant differences in HLA expression and some infiltrate markers were seen between tumors with D3 or M3. 
Table 1. Comparison of 59 patients for whom HLA expression (mRNA), chromosome 3 status, and GNAQ/GNA11 status was known. Significantly different values are represented in bold. The categorical groups were compared using a chi-squared test and numerical data were compared using an independent $t$-test.

\begin{tabular}{|c|c|c|c|c|c|c|c|}
\hline Characteristics & D3 GNA11 $(n=9)$ & D3 GNAQ $(n=12)$ & M3 GNA11 $(n=23)$ & M3 GNAQ $(n=15)$ & $\begin{array}{l}\text { Disomy } 3 \text { Tumors, GNA11 } \\
\text { versus GNAQ } p \text {-Value }\end{array}$ & $\begin{array}{l}\text { Monosomy } 3 \text { Tumors, GNA11 } \\
\text { versus GNAQ } p \text {-Value }\end{array}$ & $\begin{array}{l}\text { Monosomy } 3 \text { versus } \\
\text { Disomy } 3 p \text {-Value }\end{array}$ \\
\hline \multicolumn{8}{|l|}{ Gender $n(\%)$} \\
\hline Female & $3(33)$ & $5(42)$ & $13(57)$ & $8(53)$ & $0.70^{1}$ & $0.85^{1}$ & $0.21^{1}$ \\
\hline Male & $6(66)$ & $7(58)$ & $10(44)$ & $7(47)$ & & & \\
\hline \multicolumn{8}{|l|}{ Survival $n(\%)$} \\
\hline Alive & $7(78)$ & $3(25)$ & $3(13)$ & $2(13)$ & \multirow{4}{*}{$0.11^{1}$} & \multirow{4}{*}{$0.86^{1}$} & \multirow{4}{*}{$0.001^{1}$} \\
\hline Cause of Death: & & & & & & & \\
\hline $\begin{array}{l}\text { Melanoma-related } \\
\text { Different cause }\end{array}$ & $\begin{array}{l}1(11) \\
1(11)\end{array}$ & $\begin{array}{l}4(33) \\
3(25)\end{array}$ & $\begin{array}{c}18(78) \\
1(4)\end{array}$ & $\begin{array}{c}12(80) \\
1(7)\end{array}$ & & & \\
\hline $\begin{array}{l}\text { Dirferent cause } \\
\text { Unknown }\end{array}$ & $\begin{array}{l}1(11) \\
0(0)\end{array}$ & $2(17)$ & $1(4)$ & $0(0)$ & & & \\
\hline \multicolumn{8}{|l|}{ Affected eye $n(\%)$} \\
\hline Right eye & $3(33)$ & $5(42)$ & $13(57)$ & $7(47)$ & \multirow[t]{2}{*}{$0.70^{1}$} & \multirow{2}{*}{$0.55^{1}$} & \multirow[t]{2}{*}{$0.28^{1}$} \\
\hline Left eye & $6(66)$ & $7(58)$ & $10(44)$ & $8(53)$ & & & \\
\hline \multicolumn{5}{|l|}{ Age at enucleation } & \multirow{3}{*}{$0.14^{2}$} & \multirow{3}{*}{$0.65^{2}$} & \multirow{3}{*}{$0.012^{2}$} \\
\hline Mean & 47.24 & 59.11 & 63.95 & 65.91 & & & \\
\hline \pm St. dev. & \pm 17.82 & \pm 16.75 & \pm 15.28 & \pm 10.67 & & & \\
\hline \multicolumn{5}{|l|}{ Metastases $n(\%)$} & \multirow{3}{*}{$0.24^{1}$} & \multirow{3}{*}{$0.90^{1}$} & \multirow{3}{*}{$<0.001^{1}$} \\
\hline No & $8(89)$ & $8(67)$ & $5(22)$ & $3(20)$ & & & \\
\hline Yes & 1 (11) & $4(33)$ & $18(78)$ & $12(80)$ & & & \\
\hline \multicolumn{5}{|l|}{ Cell type $n(\%)$} & \multirow{3}{*}{$0.43^{1}$} & \multirow{3}{*}{$0.78^{1}$} & \multirow{3}{*}{$0.04^{1}$} \\
\hline Spindle & $5(56)$ & $6(50)$ & $4(17)$ & $4(26)$ & & & \\
\hline $\begin{array}{c}\text { Mixed } \\
\text { Epithelioid }\end{array}$ & $3(33)$ & $\begin{array}{l}6(50) \\
0(0)\end{array}$ & $15(65)$ & $9(60)$ & & & \\
\hline \multicolumn{5}{|l|}{ Basal diameter } & \multirow{3}{*}{$0.20^{2}$} & \multirow{3}{*}{$0.08^{2}$} & \\
\hline Mean $(\mathrm{mm})$ & 12.00 & 13.42 & 13.13 & 15.67 & & & $0.18^{2}$ \\
\hline \pm St. dev. & \pm 1.80 & \pm 3.09 & \pm 3.29 & \pm 4.62 & & & \\
\hline Prominence & & & & & & & \\
\hline Mean $(m m)$ & 7.00 & 7.29 & 7.85 & 8.13 & $0.81^{2}$ & $0.75^{2}$ & $0.30^{2}$ \\
\hline \pm St. dev. & \pm 2.18 & \pm 3.39 & \pm 2.89 & \pm 2.59 & & & \\
\hline Mitotic count & & & & & & & \\
\hline Mean $^{3}$ & 9.00 & 4.58 & 5.52 & 9.29 & $0.07^{2}$ & $0.13^{2}$ & $0.76^{2}$ \\
\hline \pm St. Dev. & \pm 5.83 & \pm 3.77 & \pm 3.40 & \pm 8.33 & & & \\
\hline Ciliary body involveme & & & & & & & \\
\hline$n(\%)$ & & & & & $0.72^{1}$ & $0.58^{1}$ & $0.009^{1}$ \\
\hline $\begin{array}{l}\text { No } \\
\text { Yes }\end{array}$ & $\begin{array}{l}8(89) \\
1(11)\end{array}$ & $\begin{array}{l}10(83) \\
2(17)\end{array}$ & $\begin{array}{l}11(48) \\
12(52)\end{array}$ & $\begin{array}{l}\begin{array}{l}(577) \\
6(43)\end{array} \\
-\end{array}$ & & & \\
\hline Extra-ocular extension & & & & & & & \\
\hline$<5 \mathrm{~mm}$ & $0(0)$ & $1(50)$ & $4(75)$ & $0(0)$ & - & $0.12^{1}$ & $0.67^{1}$ \\
\hline$>5 \mathrm{~mm}$ & $0(0)$ & $1(50)$ & 1 (25) & $1(100)$ & & & \\
\hline Chr. 8q status $n(\%)$ & & & & & & & \\
\hline Normal & $7(78)$ & $5(42)$ & $4(17)$ & $1(7)$ & $0.10^{1}$ & $0.34^{1}$ & $<0.001^{1}$ \\
\hline Aberrant & $2(22)$ & $7(58)$ & $19(83)$ & $14(93)$ & & & \\
\hline Chr. 6p status $n(\%)$ & & & & & & & \\
\hline Normal & $2(22)$ & $1(8)$ & $20(87)$ & $12(80)$ & $0.37^{1}$ & $0.57^{1}$ & $<0.001^{1}$ \\
\hline Aberrant & $7(78)$ & $11(92)$ & $3(13)$ & $3(20)$ & & & \\
\hline
\end{tabular}

${ }^{1}$ Pearson chi-squared test. ${ }^{2}$ Two-tailed $t$-test (without equal variances). ${ }^{3}$ mitosis $/ 2 \mathrm{~mm}^{2}$. Evaluating the group sizes in a Fisher's exact test yielded no significance either ( $p$-value $\left.=0.094\right)$. This means that the ratio of patients with M3/D3 is not significantly different between patients that have a mutation on a different gene. 
Table 2. Comparison of 59 patients for whom HLA expression (mRNA), chromosome 3 status, and type of GNAQ/GNA11 mutation was known. Significant values are shown in bold. The categorical groups were compared using a chi-squared test and numerical data was compared using an independent $t$-test.

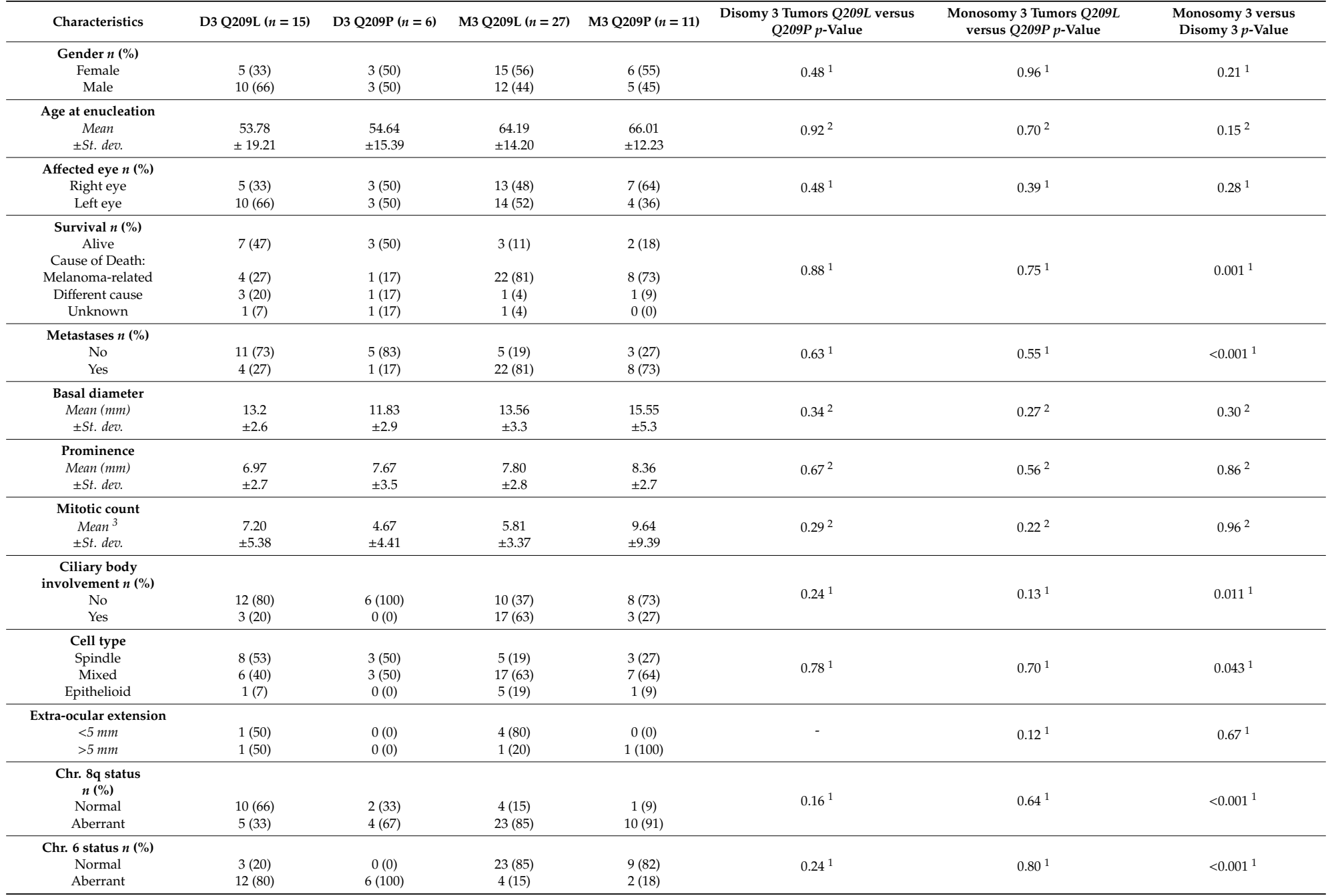

${ }^{1}$ Pearson's chi-squared test. ${ }^{2}$ Two-tailed $t$-test (without equal variances). ${ }^{3}$ mitosis/ $2 \mathrm{~mm}^{2}$. Evaluating the group sizes for the different groups in Table 2 with a Fisher's exact test yielded no significance either $(p$-value $=0.235)$. This means that the ratio of M3/D3 patients is not significantly different between the type of mutation. 
Table 3. Mean expression of mRNA expression of HLA Class I antigens and infiltrating immune cells in relation to the GNA11 or GNAQ mutation. $p$-values were calculated using the Wilcoxon-Rank sum test. STD = standard deviation.

\begin{tabular}{|c|c|c|c|c|c|c|c|c|}
\hline $\begin{array}{l}\text { mRNA } \\
\text { Probe }\end{array}$ & Illumina & $\begin{array}{c}\text { D3 } \\
\text { GNA11 } \\
n=9 \\
\text { Mean } \pm \text { STD } \\
\end{array}$ & $\begin{array}{c}\text { D3 } \\
\text { GNAQ } \\
n=12 \\
\text { Mean } \pm \text { STD } \\
\end{array}$ & $\begin{array}{c}\text { M3 } \\
\text { GNA11 } \\
n=23 \\
\text { Mean } \pm \text { STD } \\
\end{array}$ & $\begin{array}{c}\text { M3 } \\
\text { GNAQ } \\
n=15 \\
\text { Mean } \pm \text { STD } \\
\end{array}$ & $\begin{array}{l}\text { M3 GNA11 versus } \\
\text { M3 GNAQ } p \text {-Value }\end{array}$ & $\begin{array}{l}\text { D3 GNA11 versus } \\
\text { D3 GNAQ } p \text {-Value }\end{array}$ & $\begin{array}{c}\text { All M3 versus } \\
\text { All D3 } \\
p \text {-Value } \\
\end{array}$ \\
\hline HLA_A & $\begin{array}{c}\text { ILMN } \\
1671054\end{array}$ & $\begin{array}{l}10.69 \\
\pm 0.74\end{array}$ & $\begin{array}{l}10.89 \\
\pm 0.73\end{array}$ & $\begin{array}{l}11.85 \\
\pm 0.84\end{array}$ & $\begin{array}{l}11.67 \\
\pm 0.68\end{array}$ & 0.30 & 0.34 & $<0.001$ \\
\hline HLA_A & $\begin{array}{l}\text { ILMN } \\
2203950\end{array}$ & $\begin{array}{l}13.16 \\
\pm 0.96\end{array}$ & $\begin{array}{l}13.38 \\
\pm 0.64\end{array}$ & $\begin{array}{l}14.26 \\
\pm 0.53\end{array}$ & $\begin{array}{l}14.13 \\
\pm 0.49\end{array}$ & 0.42 & 0.13 & $<0.001$ \\
\hline HLA_A & $\begin{array}{l}\text { ILMN } \\
2186806\end{array}$ & $\begin{array}{c}9.87 \\
\pm 2.08\end{array}$ & $\begin{array}{c}9.89 \\
\pm 0.89\end{array}$ & $\begin{array}{l}11.31 \\
\pm 1.07\end{array}$ & $\begin{array}{l}11.13 \\
\pm 1.22\end{array}$ & 0.79 & 0.27 & $<0.001$ \\
\hline HLA_B & $\begin{array}{c}\text { ILMN } \\
1778401\end{array}$ & $\begin{array}{l}10.25 \\
\pm 2.24\end{array}$ & $\begin{array}{l}10.18 \\
\pm 1.09\end{array}$ & $\begin{array}{l}11.98 \\
\pm 1.40\end{array}$ & $\begin{array}{l}12.05 \\
\pm 1.10\end{array}$ & 0.95 & 0.21 & $<0.001$ \\
\hline HLA_C & $\begin{array}{l}\text { ILMN } \\
2150787\end{array}$ & $\begin{array}{c}6.57 \\
\pm 0.74 \\
\end{array}$ & $\begin{array}{r}6.22 \\
\pm 0.41 \\
\end{array}$ & $\begin{array}{c}6.52 \\
\pm 0.64 \\
\end{array}$ & $\begin{array}{r}6.53 \\
\pm 0.75 \\
\end{array}$ & 0.46 & 0.21 & 0.38 \\
\hline HLA_C & $\begin{array}{l}\text { ILMN } \\
1721113\end{array}$ & $\begin{array}{c}7.63 \\
\pm 1.18\end{array}$ & $\begin{array}{c}7.46 \\
\pm 0.53\end{array}$ & $\begin{array}{r}8.20 \\
\pm 1.21\end{array}$ & $\begin{array}{r}8.35 \\
\pm 1.13\end{array}$ & 0.70 & 0.80 & 0.013 \\
\hline HLA_E & $\begin{array}{l}\text { ILMN } \\
1765258\end{array}$ & $\begin{array}{l}11.10 \\
\pm 1.52\end{array}$ & $\begin{array}{l}10.84 \\
\pm 0.59\end{array}$ & $\begin{array}{l}11.83 \\
\pm 1.11\end{array}$ & $\begin{array}{l}11.73 \\
\pm 0.85\end{array}$ & 0.74 & 0.75 & $<0.001$ \\
\hline HLA_F & $\begin{array}{c}\text { ILMN } \\
1762861\end{array}$ & $\begin{array}{c}7.65 \\
\pm 1.18\end{array}$ & $\begin{array}{c}7.65 \\
\pm 0.70\end{array}$ & $\begin{array}{l}8.64 \\
\pm 1.17\end{array}$ & $\begin{array}{l}8.46 \\
\pm 0.97\end{array}$ & 0.68 & 0.59 & $<0.001$ \\
\hline HLA_G & $\begin{array}{l}\text { ILMN } \\
1656670\end{array}$ & $\begin{array}{c}8.25 \\
\pm 1.48\end{array}$ & $\begin{array}{l}8.08 \\
\pm 0.64\end{array}$ & $\begin{array}{l}9.05 \\
\pm 0.76\end{array}$ & $\begin{array}{l}8.88 \\
\pm 0.70\end{array}$ & 0.39 & 0.70 & $<0.001$ \\
\hline CD68 & $\begin{array}{c}\text { ILMN } \\
1714861\end{array}$ & $\begin{array}{r}10.36 \\
\pm 1.30\end{array}$ & $\begin{array}{l}10.37 \\
\pm 0.86\end{array}$ & $\begin{array}{l}11.07 \\
\pm 0.75\end{array}$ & $\begin{array}{l}11.01 \\
\pm 0.82\end{array}$ & 0.95 & 0.70 & 0.008 \\
\hline CD3D & $\begin{array}{l}\text { ILMN } \\
2261416\end{array}$ & $\begin{array}{c}7.07 \\
\pm 1.70\end{array}$ & $\begin{array}{c}6.66 \\
\pm 0.40\end{array}$ & $\begin{array}{c}7.28 \\
\pm 1.12\end{array}$ & $\begin{array}{c}7.28 \\
\pm 1.16\end{array}$ & 0.95 & 0.92 & 0.03 \\
\hline CD3D & $\begin{array}{l}\text { ILMN } \\
2325837\end{array}$ & $\begin{array}{l}7.14 \\
\pm 1.71\end{array}$ & $\begin{array}{c}6.57 \\
\pm 0.43\end{array}$ & $\begin{array}{c}7.37 \\
\pm 1.23\end{array}$ & $\begin{array}{c}7.31 \\
\pm 1.16\end{array}$ & 0.98 & 0.46 & 0.022 \\
\hline CD3E & $\begin{array}{c}\text { ILMN } \\
1739794\end{array}$ & $\begin{array}{c}6.56 \\
\pm 0.50\end{array}$ & $\begin{array}{c}6.38 \\
\pm 0.07\end{array}$ & $\begin{array}{c}6.51 \\
\pm 0.28\end{array}$ & $\begin{array}{c}6.57 \\
\pm 0.31\end{array}$ & 0.55 & 0.50 & 0.19 \\
\hline CD3G & $\begin{array}{c}\text { ILMN } \\
1717197\end{array}$ & $\begin{array}{c}6.68 \\
\pm 0.45\end{array}$ & $\begin{array}{c}6.53 \\
\pm 0.11 \\
\end{array}$ & $\begin{array}{c}6.63 \\
\pm 0.28\end{array}$ & $\begin{array}{c}6.57 \\
\pm 0.29\end{array}$ & 0.39 & 0.64 & 0.77 \\
\hline CD8A & $\begin{array}{c}\text { ILMN } \\
1768482\end{array}$ & $\begin{array}{c}7.30 \\
\pm 2.06\end{array}$ & $\begin{array}{c}6.78 \\
\pm 0.45\end{array}$ & $\begin{array}{l}7.45 \\
\pm 1.23\end{array}$ & $\begin{array}{c}7.54 \\
\pm 1.29\end{array}$ & 0.86 & 0.86 & 0.06 \\
\hline CD8A & $\begin{array}{l}\text { ILMN } \\
1760374\end{array}$ & $\begin{array}{c}6.58 \\
\pm 0.58\end{array}$ & $\begin{array}{c}6.36 \\
\pm 0.10\end{array}$ & $\begin{array}{c}6.47 \\
\pm 0.29\end{array}$ & $\begin{array}{l}6.54 \\
\pm 0.45\end{array}$ & 0.63 & 0.27 & 0.25 \\
\hline CD8A & $\begin{array}{l}\text { ILMN } \\
2353732\end{array}$ & $\begin{array}{c}7.22 \\
\pm 2.11\end{array}$ & $\begin{array}{c}6.65 \\
\pm 0.43\end{array}$ & $\begin{array}{r}7.42 \\
\pm 1.25\end{array}$ & $\begin{array}{c}7.46 \\
\pm 1.31\end{array}$ & 0.98 & 0.80 & 0.022 \\
\hline CD4 & $\begin{array}{l}\text { ILMN } \\
1727284\end{array}$ & $\begin{array}{l}6.65 \\
\pm 0.49\end{array}$ & $\begin{array}{c}6.54 \\
\pm 0.29\end{array}$ & $\begin{array}{c}6.69 \\
\pm 0.24\end{array}$ & $\begin{array}{c}6.73 \\
\pm 0.36\end{array}$ & 0.98 & 0.86 & 0.044 \\
\hline
\end{tabular}


Table 4. Mean expression of mRNA expression of HLA Class I antigens and infiltrating immune cells in relation to Q209L or Q209P mutation on either GNAQ or GNA11. $p$-values were calculated using the Wilcoxon-Rank sum test. STD = standard deviation.

\begin{tabular}{|c|c|c|c|c|c|c|c|c|}
\hline $\begin{array}{l}\text { mRNA } \\
\text { Probe }\end{array}$ & Illumina & $\begin{array}{c}\text { D3 } \\
\text { Q209L } \\
n=15 \\
\text { Mean } \pm \text { STD }\end{array}$ & $\begin{array}{c}\text { D3 } \\
\text { Q209P } \\
n=6 \\
\text { Mean } \pm \text { STD }\end{array}$ & $\begin{array}{c}\text { M3 } \\
\text { Q209L } \\
n=27 \\
\text { Mean } \pm \text { STD }\end{array}$ & $\begin{array}{c}\text { M3 } \\
\text { Q209P } \\
n=11 \\
\text { Mean } \pm \text { STD }\end{array}$ & $\begin{array}{c}\text { M3 Q209L versus M3 } \\
\text { Q209P } \\
p \text {-Value }\end{array}$ & $\begin{array}{c}\text { D3 Q209L versus D3 } \\
\text { Q209P } \\
p \text {-Value }\end{array}$ & $\begin{array}{c}\text { All M3 versus All D3 } \\
p \text {-Value }\end{array}$ \\
\hline HLA_A & $\begin{array}{c}\text { ILMN } \\
1671054\end{array}$ & $\begin{array}{l}10.88 \\
\pm 0.73\end{array}$ & $\begin{array}{l}10.63 \\
\pm 0.72\end{array}$ & $\begin{array}{l}11.85 \\
\pm 0.79\end{array}$ & $\begin{array}{l}11.60 \\
\pm 0.74\end{array}$ & 0.30 & 0.79 & $<0.001$ \\
\hline HLA_A & $\begin{array}{c}\text { ILMN } \\
2203950\end{array}$ & $\begin{array}{l}13.35 \\
\pm 0.80\end{array}$ & $\begin{array}{l}13.12 \\
\pm 0.75\end{array}$ & $\begin{array}{l}14.23 \\
\pm 0.51\end{array}$ & $\begin{array}{l}14.16 \\
\pm 0.55\end{array}$ & 0.87 & 0.91 & $<0.001$ \\
\hline HLA_A & $\begin{array}{c}\text { ILMN } \\
2186806\end{array}$ & $\begin{array}{l}10.08 \\
\pm 1.63\end{array}$ & $\begin{array}{c}9.39 \\
\pm 0.93\end{array}$ & $\begin{array}{l}11.20 \\
\pm 1.10\end{array}$ & $\begin{array}{l}11.33 \\
\pm 1.22\end{array}$ & 0.70 & 0.37 & $<0.001$ \\
\hline HLA_B & $\begin{array}{c}\text { ILMN } \\
1778401\end{array}$ & $\begin{array}{l}10.34 \\
\pm 1.78\end{array}$ & $\begin{array}{c}9.89 \\
\pm 1.26 \\
\end{array}$ & $\begin{array}{l}11.99 \\
\pm 1.34\end{array}$ & $\begin{array}{l}12.05 \\
\pm 1.17\end{array}$ & 1.00 & 0.97 & $<0.001$ \\
\hline HLA_C & $\begin{array}{c}\text { ILMN } \\
2150787\end{array}$ & $\begin{array}{c}6.34 \\
\pm 0.66\end{array}$ & $\begin{array}{l}6.44 \\
\pm 0.37\end{array}$ & $\begin{array}{c}6.44 \\
\pm 0.63\end{array}$ & $\begin{array}{l}6.74 \\
\pm 0.77\end{array}$ & 0.46 & 0.51 & 0.38 \\
\hline HLA_C & $\begin{array}{c}\text { ILMN } \\
1721113\end{array}$ & $\begin{array}{l}7.57 \\
\pm 0.95\end{array}$ & $\begin{array}{c}7.43 \\
\pm 0.58\end{array}$ & $\begin{array}{c}8.16 \\
\pm 1.19\end{array}$ & $\begin{array}{c}8.50 \\
\pm 1.12\end{array}$ & 0.42 & 0.97 & 0.013 \\
\hline HLA_E & $\begin{array}{c}\text { ILMN } \\
1765258\end{array}$ & $\begin{array}{l}11.01 \\
\pm 1.21\end{array}$ & $\begin{array}{l}10.81 \\
\pm 0.61\end{array}$ & $\begin{array}{l}11.82 \\
\pm 1.05\end{array}$ & $\begin{array}{l}11.71 \\
\pm 0.93\end{array}$ & 0.63 & 1.00 & $<0.001$ \\
\hline HLA_F & $\begin{array}{c}\text { ILMN } \\
1762861\end{array}$ & $\begin{array}{c}7.72 \\
\pm 1.00\end{array}$ & $\begin{array}{r}7.49 \\
\pm 0.69\end{array}$ & $\begin{array}{l}8.58 \\
\pm 1.13\end{array}$ & $\begin{array}{l}8.54 \\
\pm 1.02\end{array}$ & 1.00 & 0.67 & $<0.001$ \\
\hline HLA_G & $\begin{array}{c}\text { ILMN } \\
1656670\end{array}$ & $\begin{array}{c}8.32 \\
\pm 1.16\end{array}$ & $\begin{array}{l}7.73 \\
\pm 0.61\end{array}$ & $\begin{array}{c}9.00 \\
\pm 0.76\end{array}$ & $\begin{array}{c}8.96 \\
\pm 0.69\end{array}$ & 0.85 & 0.15 & $<0.001$ \\
\hline CD68 & $\begin{array}{c}\text { ILMN } \\
1714861\end{array}$ & $\begin{array}{l}10.39 \\
\pm 1.16\end{array}$ & $\begin{array}{l}10.30 \\
\pm 0.75\end{array}$ & $\begin{array}{l}11.07 \\
\pm 0.74\end{array}$ & $\begin{array}{l}10.99 \\
\pm 0.87\end{array}$ & 0.80 & 0.85 & 0.008 \\
\hline CD3D & $\begin{array}{c}\text { ILMN } \\
2261416\end{array}$ & $\begin{array}{c}6.92 \\
\pm 1.32\end{array}$ & $\begin{array}{c}6.61 \\
\pm 0.45\end{array}$ & $\begin{array}{c}7.23 \\
\pm 1.06\end{array}$ & $\begin{array}{c}7.41 \\
\pm 1.29\end{array}$ & 0.85 & 0.51 & 0.033 \\
\hline CD3D & $\begin{array}{c}\text { ILMN } \\
2325837\end{array}$ & $\begin{array}{l}6.94 \\
\pm 1.39\end{array}$ & $\begin{array}{c}6.49 \\
\pm 0.47\end{array}$ & $\begin{array}{c}7.31 \\
\pm 1.17\end{array}$ & $\begin{array}{c}7.44 \\
\pm 1.29\end{array}$ & 0.75 & 0.23 & 0.016 \\
\hline CD3E & $\begin{array}{c}\text { ILMN } \\
1739794\end{array}$ & $\begin{array}{c}6.49 \\
\pm 0.39\end{array}$ & $\begin{array}{c}6.37 \\
\pm 0.09\end{array}$ & $\begin{array}{c}6.51 \\
\pm 0.26\end{array}$ & $\begin{array}{l}6.60 \\
\pm 0.35\end{array}$ & 0.56 & 0.41 & 0.19 \\
\hline CD3G & $\begin{array}{l}\text { ILMN } \\
1717197\end{array}$ & $\begin{array}{c}6.60 \\
\pm 0.36\end{array}$ & $\begin{array}{c}6.58 \\
\pm 0.13\end{array}$ & $\begin{array}{c}6.61 \\
\pm 0.27\end{array}$ & $\begin{array}{c}6.60 \\
\pm 0.33\end{array}$ & 0.70 & 0.51 & 0.77 \\
\hline CD8A & $\begin{array}{c}\text { ILMN } \\
1768482\end{array}$ & $\begin{array}{l}7.10 \\
\pm 1.59\end{array}$ & $\begin{array}{c}6.75 \\
\pm 0.58 \\
\end{array}$ & $\begin{array}{c}7.39 \\
\pm 1.17\end{array}$ & $\begin{array}{c}7.71 \\
\pm 1.43\end{array}$ & 0.56 & 0.67 & 0.061 \\
\hline CD8A & $\begin{array}{c}\text { ILMN } \\
1760374\end{array}$ & $\begin{array}{c}6.50 \\
\pm 0.46\end{array}$ & $\begin{array}{c}6.35 \\
\pm 0.09\end{array}$ & $\begin{array}{c}6.47 \\
\pm 0.27\end{array}$ & $\begin{array}{c}6.57 \\
\pm 0.52\end{array}$ & 0.80 & 0.41 & 0.25 \\
\hline CD8A & $\begin{array}{c}\text { ILMN } \\
2353732\end{array}$ & $\begin{array}{c}7.00 \\
\pm 1.63\end{array}$ & $\begin{array}{c}6.62 \\
\pm 0.55\end{array}$ & $\begin{array}{c}7.35 \\
\pm 1.19\end{array}$ & $\begin{array}{c}7.64 \\
\pm 1.44\end{array}$ & 0.52 & 0.46 & 0.019 \\
\hline CD4 & $\begin{array}{c}\text { ILMN } \\
1727284\end{array}$ & $\begin{array}{c}6.62 \\
\pm 0.41\end{array}$ & $\begin{array}{c}6.51 \\
\pm 0.21\end{array}$ & $\begin{array}{c}6.70 \\
\pm 0.25\end{array}$ & $\begin{array}{l}6.72 \\
\pm 0.40\end{array}$ & 0.90 & 0.61 & 0.036 \\
\hline
\end{tabular}




\subsection{Survival}

Additionally, the survival of the different patient-groups was examined. A difference in survival could indicate a difference between the tumors that is not seen using these mRNA probes (Figure 2a,b). In Figure 2a,b there is no significant difference between the GNA11/GNAQ or Q209L/Q209P mutations. However, as expected, the data shows a significant association with M3 status.

\section{Kaplan-Meier analysis of patients based on mutated gene}

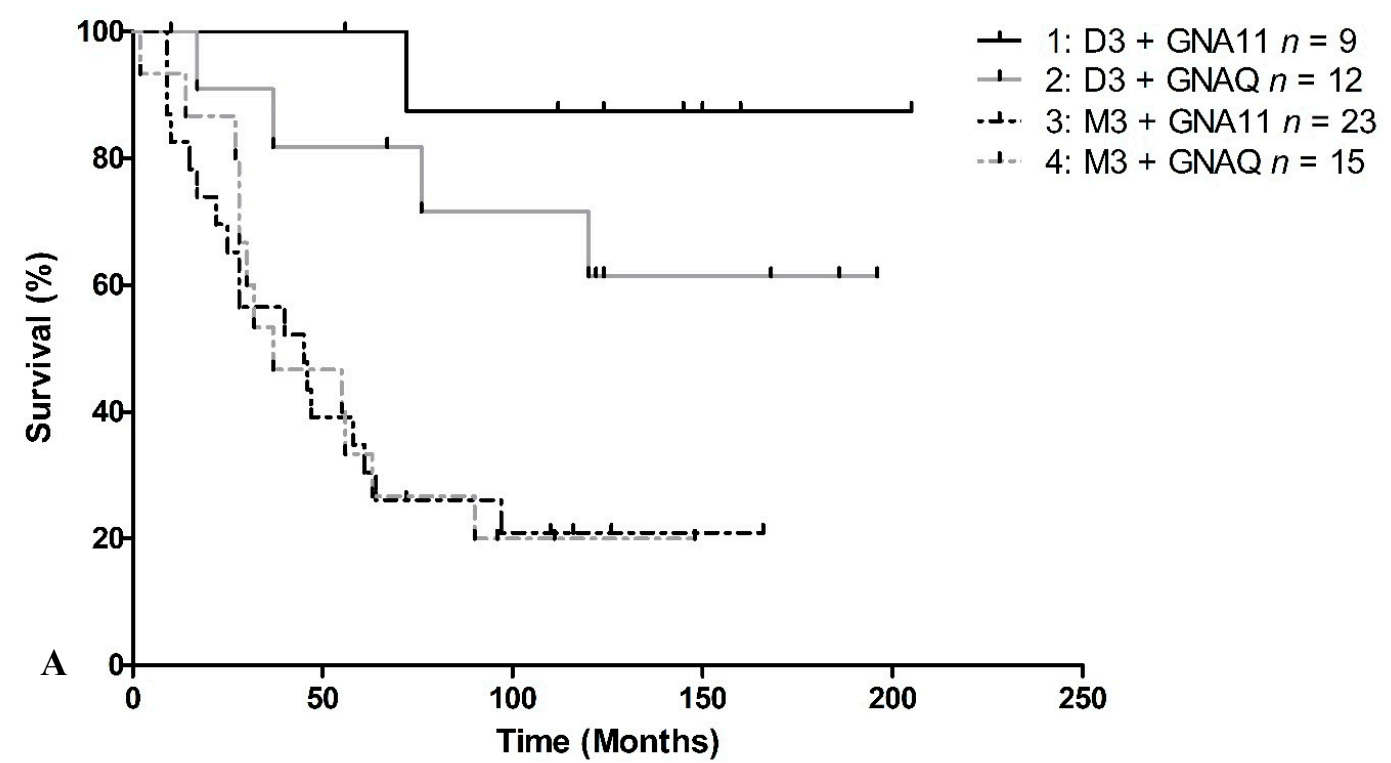

Kaplan-Meier analysis of patients based on Q209 mutation

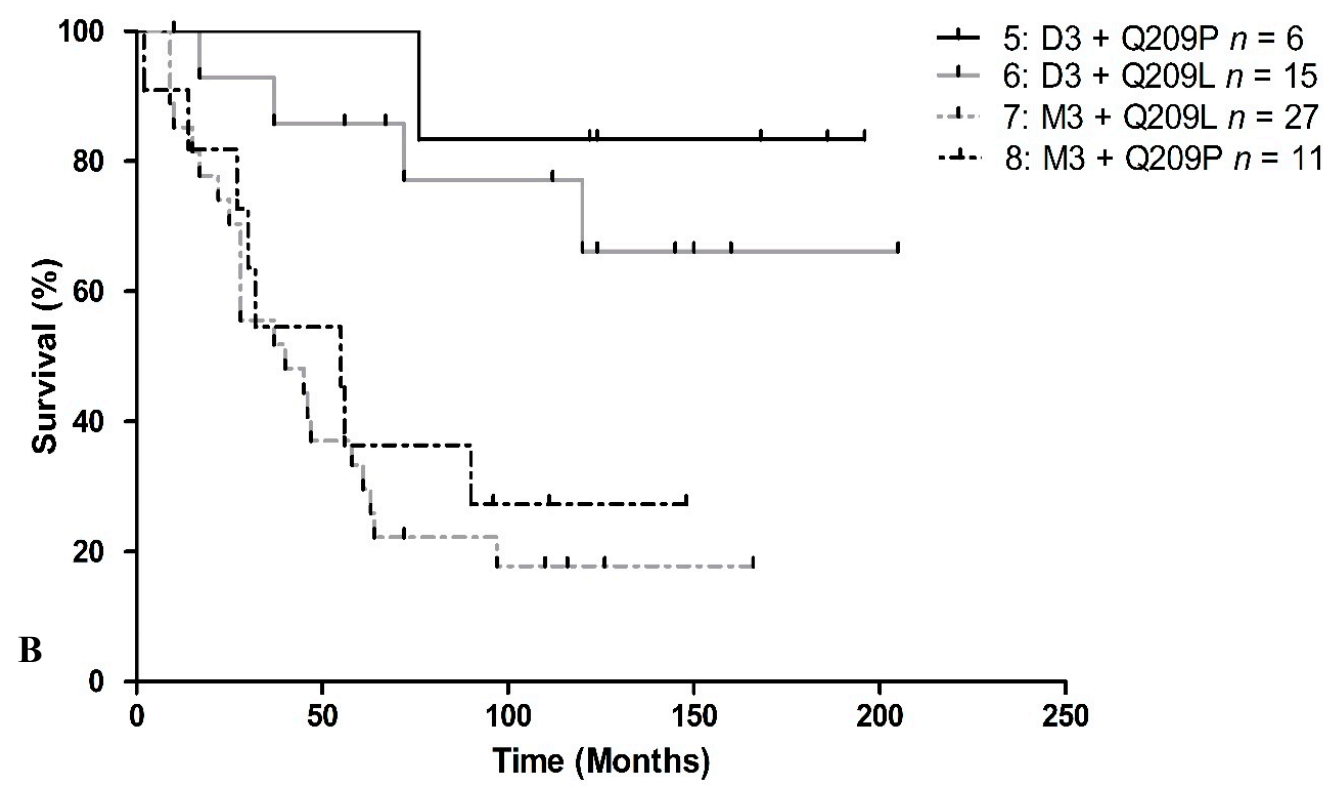

Figure 2. Survival of 59 UM patients, split depending on the location of the mutation (A) or type of mutation (B). $p$-values for the differences between the groups calculated with the log-rank function were (A): 1 versus 2: $p=0.47, \mathbf{1}$ versus 3: $p<0.001,2$ versus 4: $p=0.030,3$ versus 4: $p=0.90$; (B): 5 versus $6: p=0.77,6$ versus $7: p=0.002,5$ versus $7: p=0.007,7$ versus $8: p=0.07$. 


\subsection{HLA Expression in Relation to Chromosome 8q Status}

Instead of a GNAQ/11 mutation, a difference in chromosome 6 or $8 \mathrm{q}$ status might influence HLA expression. Gezgin has shown that the presence of macrophages is associated with 8q [28]. While almost all M3 tumors also have additional copies of 8q, there is variety between D3 tumors. However, there is no significant difference in HLA expression between D3 tumors with or without chromosome 8q aberrations (Figure 3).

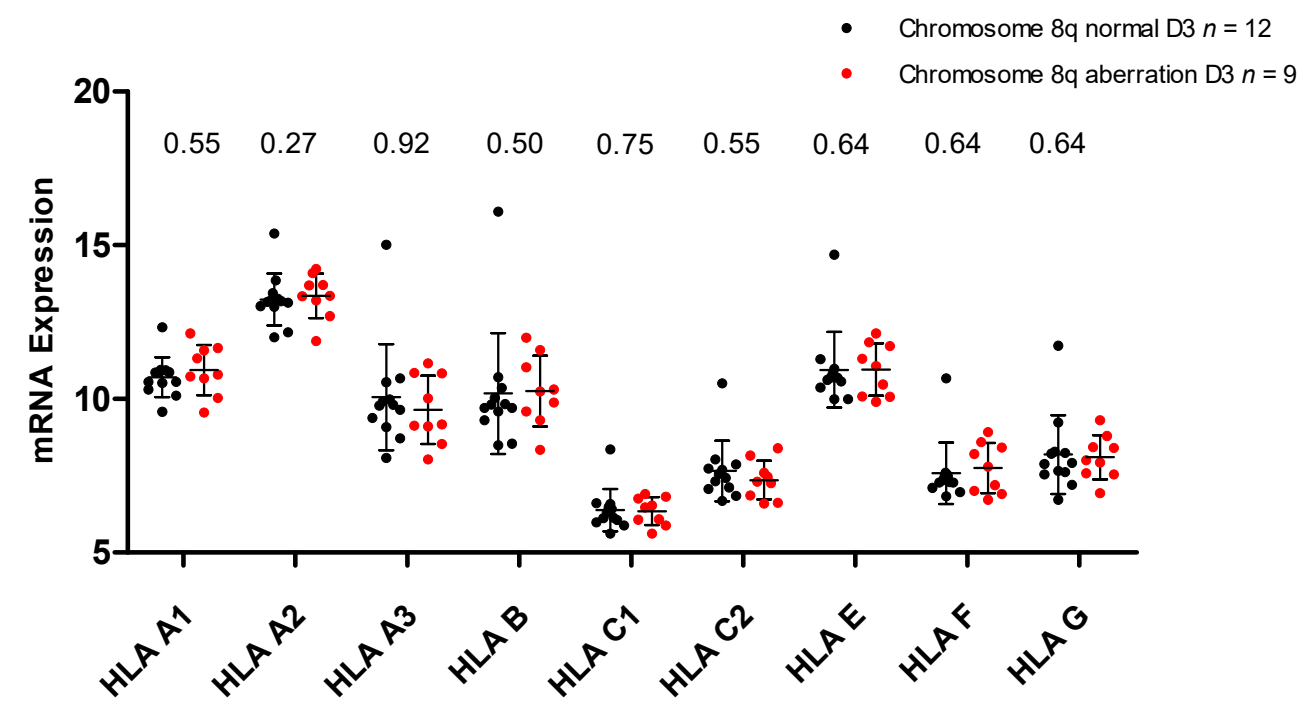

Figure 3. mRNA expression of all D3 tumors split on chromosome 8q status, shown for multiple probes ( $p$-values shown were obtained using the Mann-Whitney U-test, comparing D3/chr8q normal $(n=12)$ with D3/Chr8q aberrant tumors $(n=9))$.

\section{Discussion}

Despite the small group sizes for GNA11 and GNAQ tumors it is quite clear that there is no significant difference in the expression patterns of HLA Class I and the presence of T cells or macrophages between tumors with either a GNAQ or GNA11 mutation or a Q209P or Q209P mutation. Therefore, there is no evidence that the differences in inflammation found in groups with the same chromosome 3 status are caused by the different mutations on GNAQ or GNA11 or the type of mutation Q209L or Q209P. The literature also suggests mutations on GNAQ do not affect the survival, and this was confirmed in this study, as no difference between GNAQ/GNA11 was observed [30]. Due to Q209 being crucial for GTPase activity, GTP hydrolysis is abolished in both types of mutation in both genes [31]. As we did not find any difference, there is probably no difference in any pathway activation between the different mutations, or any different secondary effect of the sub-units formed from GNA11 and GNAQ. If a specific mutation would have changed the protein into one that remained functional but with less affinity, the different mutations could have explained the difference in inflammation. We did not further study any relation with the Yes-Associated Protein (YAP) pathway as we did not find any differences related to the $G N A Q / 11$ mutations.

M3 and D3 tumors showed a significant difference in expression with regard to most $\mathrm{T}$ cell and macrophage probes. This was expected based on previous research [23,28,32]. However, we now show that the expression of all the different HLA Class I molecules is related to the tumor's chromosome 3 status. However, both within the D3 group as well as within the M3 group, some tumors were observed to have more inflammation than others, indicating that there must also be other factors that have yet to be identified, besides the chromosome aberrations.

However, our data indicates that GNAQ and GNA11 do not play a direct role in regulating inflammation. This is reflected in Figure 4. If the heatmap from Figure 1 is rearranged according 
to the GNAQ/GNA11 mutation status, no obvious difference is seen between the GNA11-mutated tumors on the left and the GNAQ-mutated tumors on the right. Previous work on GNAQ and GNA11 has shown that they activate the G protein signaling cascade, leading to the stimulation of Mitogen-Activated Protein (MAP) kinases, protein kinase B (Akt), protein kinase C (PKC), and Rho GTPase $[33,34]$. Blocking of PKC activity has been associated with downregulation of the nuclear factor kappa-light-chain-enhancer of activated B cells (NFKB) pathway, one of the leading regulators of HLA expression [35,36], while Rho GTPase has been associated with HIF1a upregulation, also leading to NFKB activity [37]. As we saw differences in inflammation between tumors with the same chromosome 3 status, we considered the option that the two types of mutations might differentially affect this inflammation. Although there was no difference, it is quite possible that both mutations similarly stimulate an inflammatory environment in melanocytes, such as nevi [5].

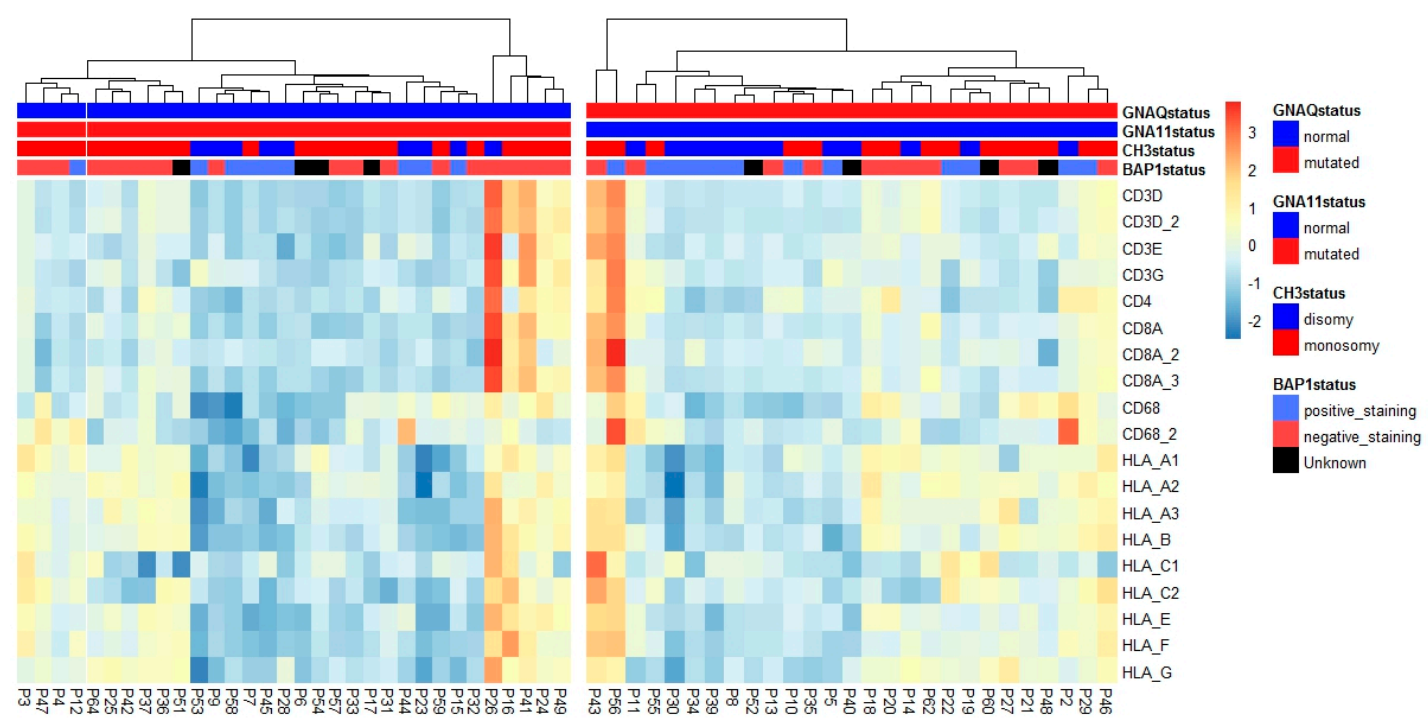

Figure 4. Heatmap of tumors split according to GNAQ/GNA11 mutation. The heatmap shows the expression of several infiltrate and HLA Class I markers, normalized per marker over all 59 tumors. Annotated is the chromosome 3 status (blue for D3 tumors, red for M3 tumors). BAP1 staining is annotated below the chromosome 3 status. Positive staining for BAP1 is blue (BAP1 is normal), red is given for absent staining and black is given for unknown BAP1 status. Mutations on GNAQ/GNA11 only include Q209L and Q209P mutations. GNAQ mutated: $n=27$, GNA11 mutated: $n=32$.

The heatmap in Figure 4 clearly shows that several groups with different levels of inflammation and HLA expression exist in the different GNAQ or GNA11 mutated tumors. These differences are not all related to the chromosome 3 status. However, it is yet unknown what causes the cause of the inflammation within the tumors with the same chromosome 3 status.

Finding the cause of the relation between M3 and inflammation may help us to better understand how inflammation is involved in spreading metastasis.

Any differences found between the groups could be caused by other rarer secondary mutations but are not caused by a GNAQ/GNA11 mutation. We do not find a difference in survival between the tumors with different GNAQ/GNA11 mutations, while a clear difference can be observed between patients with a different chromosome 3 status. How the loss of chromosome 3/BAP1 leads to an inflammatory phenotype will be the subject of further studies. 


\section{Materials and Methods}

\subsection{Cases}

A total of 64 tumor samples were collected from enucleated eyes at the Department of Ophthalmology at the Leiden University Medical Centre (LUMC) in Leiden, the Netherlands. All tumor samples were from patients who underwent primary enucleation for UM between 1999 and 2009. The collection of materials and research protocol were compliant with the tenets of the declaration of Helsinki (World Medical Association of Declaration 2013; ethical principles for medical research involving human subjects). Tumor material was handled in accordance with the Dutch National Ethical Guidelines ('Code for Proper Secondary Use of Human Tissue").

The pathological data was obtained from patient charts. The tumors were examined by a pathologist specializing in ocular-oncology.

The Medisch Ethische Toetsingscommissie (METC) gave approval on 19 October 2016 with the code G16.076/NV/gk.

\subsection{Chromosome Analysis}

Following enucleation, small parts of the tumor were sent out for cell culturing [16]. These cells were cultured and those that were successfully cultured had karyotyping performed on them. Some of the tumors also had a FISH analysis performed on them.

Samples collected at the Leiden University Medical Center underwent DNA isolation using a QIAmp DNA Mini Kit (Qiagen, Venlo, the Netherlands) following the manufacturer's guidelines.

An SNP analysis was performed on all 64 analyzed tumors. For the SNP assay, two microarray chips were used: the Affymetrix 250K_NSP-chip, which holds approximately 250,000 probes across the genome, and the Affymetrix Cytoscan HD chip, which holds approximately 750,000 probes across the genome. The copy number was determined using the "genotyping console (GTC)" (Affymetrix, Santa Clara, CA, USA). The "GTC Browser" (Affymetrix, Santa Clara, CA, USA) was used to visualize the data of the Affymetrix 250K_NSP. The Affymetrix Cytoscan HD chips were analyzed using the "Chromosome Analysis Suite" 'ChaS' (Affymetrix). The thresholds for chromosome aberrations were: $<1.9$ loss, 1.9-2.1 normal, $>2.1<3.1$ gain, $>3.1$ amplification. In this research the loss, gain and amplifications were classified as chromosome aberrations.

In cases where karyotyping, FISH, or SNP disagreed, an abnormal situation was assumed (e.g., when karyotyping showed M3 status, even if FISH and SNP showed D3, an M3 status was assumed).

\subsection{GNAQ and GNA11 Mutations}

The presence of a mutation in either GNAQ/GNA11 was analyzed using hydrolysis probes in a duplex dPCR. Of each tumor sample $10 \mathrm{ng}$ DNA was used in a $20 \mu \mathrm{L}$ reaction volume. The protocol was performed as described before [16]. The reaction mixture consisted of $2 \times$ droplet PCR supermix (Bio-Rad Laboratories, Inc., Berkeley, CA, USA). $20 \times$ target probe (FAM), $20 \times$ wildtype probe (HEX). Proprietary probes and primers (Bio-Rad Laboratories, Inc.) were used. The following MiQE sequences:

GNAQ Q209P (ID: dHsaIS2501447 \& ID: dHsaIS2501446): TGCTATTTAAACTTGAACTCAAAGCCACCTATTTTGATACTATGTAAAAAATTATGTTGC AAACTCACACCCTAAAACTTTTTCTTTAAAGAGGTATAACTGACATACTCAGAGAGAG ATAAA

GNAQ Q209L (ID: dHsaCP2000052 \& ID: dHsaCP2000051): AGTGTATCCATTTTCTTCTCTCTGACCTTTGGCCCCCTACATCGAC CATTCTGCAAGGTTAACAATACTCATATTAATAACATATAAAGTAAA ACTAAAAAGTCAACATAAATATAGCACTAC

GNA11 209L (ID: dHsaCP2000050 \& ID: dHsaCP2000049): 


\section{GTCCTGGTGG}

Using a QX100 droplet generator and DG8 cartridges (Bio-Rad Laboratories, Inc.), each $20 \mu \mathrm{L}$ sample was converted to an emulsion of 20,000 droplets. The emulsion was then transferred to a 96-well PCR plate. The following end point PCR program was used to identify the mutations using a T100 Thermal cycler. $95^{\circ} \mathrm{C}$ for 10 minutes, then 40 cycles of: $94{ }^{\circ} \mathrm{C}$ for 30 seconds, $55^{\circ} \mathrm{C}$ for $1 \mathrm{~min}$. Followed by $98^{\circ} \mathrm{C}$ for 10 minutes and then it was kept at $4{ }^{\circ} \mathrm{C}$ until the results were read by a QX100 droplet reader (Bio-Rad Laboratories, Inc.). Digital PCR (dPCR) software (QuantaSoft, Berkeley, CA, USA) was used to read the results using fluorescence, and for analyzing the data.

\section{4. mRNA Analysis Status}

mRNA status was obtained from fresh frozen tumor tissue. RNA for gene expression profiling was isolated with the RNeasy mini kit (Qiagen). HLA Class I expression was measured on an Illumina HT-12v4 chip (Illumina) following the manufacturer's guidelines. The probes seen in Table 5 were used for this analysis. They had previously been compared with immunohistochemical data $[24,28]$.

Table 5. Probes used in the analysis.

\begin{tabular}{cc}
\hline Gene & Probe \\
\hline HLA-A & ILMN_1671054 \\
HLA-A & ILMN_2203950 \\
HLA-A & ILMN_2186806 \\
HLA-B & ILMN_1778401 \\
HLA-C & ILMN_2150787 \\
HLA-C & ILMN_1721113 \\
HLA-E & ILMN_1765258 \\
HLA-F & ILMN_1762861 \\
HLA-G & ILMN_1656670 \\
CD68 & ILMN 1714861 \\
CD3D & ILMN 2261416 \\
CD3D & ILMN 2325837 \\
CD3E & ILMN 1739794 \\
CD3G & ILMN 1717197 \\
CD8A & ILMN 1768482 \\
CD8A & ILMN 1760374 \\
CD8A & ILMN 2353732 \\
CD4 & ILMN 1727284 \\
\hline
\end{tabular}

\subsection{Statistical Analysis}

To analyze the data several programs were used. Statistical package for social sciences (SPSS) (IBM Corp; released 2015; IBM SPSS Statistics for Windows, Version 23.0; Armonk, NY, IBM Corp) was used to analyze different groups and characteristics. All analyses were done with SPSS except those regarding the HLA expression. For differences between the groups related to chromosome 3 status, a Pearson's $\chi^{2}$ test was used, and in case of more than two groups, a Fisher's exact test. When numerical data was used such as the average age at time of enucleation, a two-tailed $t$-test was used and the $p$-value that did not assume equal variances was used.

MATLAB (MATLAB and Statistics Toolbox Release 2018a, The MathWorks, Inc., Natick, Massachusetts, USA) was used to analyze the differences in HLA expression between the different groups using the Wilcoxon Rank-sum test, and to compare survival curves. For the comparison between survival curves the following file was used Cardillo G. (2008). LogRank: Comparing survival curves of two groups using the log rank test http://www.mathworks.com/matlabcentral/fileexchange/22317) version 2.0.0.0

Kaplan-Meier curves were made using GraphPad Prism version 5.00 for Windows, GraphPad Software, La Jolla California USA, www.graphpad.com. 
The analyses were performed with data of 59 tumors of which the chromosome 3 status and the mutation of GNAQ/GNA11 was known. M3 tumors were analyzed separately from D3 tumors. When separated on chromosome 3 status and GNAQ/11 mutations the following group sizes were created: D3 + GNA11 $n=9$, D3 + GNAQ $n=12$, M3 + GNA11 $n=23$, M3 + GNAQ $n=15$, D3 + Q209L $n=15$, $\mathrm{D} 3+\mathrm{Q} 209 \mathrm{P} n=6, \mathrm{M} 3+\mathrm{Q} 209 \mathrm{~L} n=27$, and M3 + Q209P $n=11$.

\section{Conclusions}

The type and location of mutations on GNAQ/GNA11 do not seem to affect the progression of UM. While these are driver mutations, there is no difference in mRNA expression of infiltrate markers and HLA Class I expression. Survival was also not significantly affected by these mutations. The chromosome 8q status did not explain HLA expression differences either. The main difference between inflamed and non-inflamed tumors is the chromosome 3 status. Chromosome 3 status also had a major influence on HLA expression. The GNAQ/GNA11 mutations do not play a major role in distinguishing between tumors with and without an inflammatory phenotype.

Author Contributions: Conceptualization, C.v.W., A.P.A.W., U.P., T.v.H., and M.J.J.; methodology, C.v.W., M.V., and P.A.v.d.V.; software, C.v.W.; validation, W.G.M.K. and M.V.; formal analysis, C.v.W.; investigation, C.v.W., A.P.A.W., and M.J.J.; resources, A.P.A.W., M.V., P.A.v.d.V., W.G.M.K., and G.P.M.L.; writing-original draft preparation, C.v.W.; writing-review and editing, A.P.A.W., M.V., T.v.H., W.M.G.K., U.P., G.P.M.L., and M.J.J.; visualization, C.v.W.; supervision, M.J.J. and A.P.A.W.; project administration, M.J.J.; funding acquisition, G.P.M.L. and M.J.J.

Funding: The research was funded by the Horizon2020 UM CURE grant \#667787 and by the IG17103 grant from the Associazione Italiana per la Ricerca sul Cancro and the Compagnia di San Paolo (\#20067) to U.P.

Conflicts of Interest: The authors declare no conflict of interest.

\section{References}

1. Virgili, G.; Gatta, G.; Ciccolallo, L.; Capocaccia, R.; Biggeri, A.; Crocetti, E.; Lutz, J.M.; Paci, E.; EUROCARE Working Group. Incidence of Uveal Melanoma in Europe. Ophthalmology 2007, 114, 2309-2315. [CrossRef] [PubMed]

2. Kujala, E.; Mäkitie, T.; Kivelä, T. Very Long-Term Prognosis of Patients with Malignant Uveal Melanoma. Investig. Ophthalmol. Vis. Sci. 2003, 44, 4651-4659. [CrossRef] [PubMed]

3. Van Raamsdonk, C.D.; Bezrookove, V.; Green, G.; Bauer, J.; Gaugler, L.; O’Brien, J.M.; Simpson, E.M.; Barsh, G.S.; Bastian, B.C. Frequent somatic mutations of GNAQ in uveal melanoma and blue naevi. Nature 2009, 457, 599-602. [CrossRef] [PubMed]

4. Van Raamsdonk, C.D.; Griewank, K.G.; Crosby, M.B.; Garrido, M.C.; Vemula, S.; Wiesner, T.; Obenauf, A.C.; Wackernagel, W.; Green, G.; Bouvier, N.; et al. Mutations in GNA11 in uveal melanoma. N. Engl. J. Med. 2010, 363, 2191-2199. [CrossRef] [PubMed]

5. Vader, M.; Madigan, M.; Versluis, M.; Suleiman, H.; Gezgin, G.; Gruis, N.A.; Out Luiting, J.J.; Bergman, W.; Verdijk, R.M.; Jager, M.J.; et al. GNAQ and GNA11 mutations and downstream YAP activation in choroidal nevi. Br. J. Cancer 2017, 117, 884-887. [CrossRef] [PubMed]

6. Harbour, J.W.; Roberson, E.D.O.; Anbunathan, H.; Onken, M.D.; Worley, L.A.; Bowcock, A.M. Recurrent mutations at codon 625 of the splicing factor SF3B1 in uveal melanoma. Nat. Genet. 2013, 45, 133-135. [CrossRef] [PubMed]

7. Martin, M.; Maßhofer, L.; Temming, P.; Rahmann, S.; Metz, C.; Bornfeld, N.; van de Nes, J.; Klein-Hitpass, L.; Hinnebusch, A.G.; Horsthemke, B.; et al. Exome sequencing identifies recurrent somatic mutations in EIF1AX and SF3B1 in uveal melanoma with disomy 3. Nat. Genet. 2013, 45, 933-936. [CrossRef] [PubMed]

8. Furney, S.J.; Pedersen, M.; Gentien, D.; Dumont, A.G.; Rapinat, A.; Desjardins, L.; Turajlic, S.; Piperno-Neumann, S.; de la Grange, P.; Roman-Roman, S.; et al. SF3B1 mutations are associated with alternative splicing in uveal melanoma. Cancer Discov. 2013, 3, 1122-1129. [CrossRef] [PubMed]

9. Prescher, G.; Bornfeld, N.; Hirche, H.; Horsthemke, B.; Jockel, K.H.; Becher, R. Prognostic implications of monosomy 3 in uveal melanoma. Lancet 1996, 347, 1222-1225. [CrossRef] 
10. Ewens, K.G.; Kanetsky, P.A.; Richards-Yutz, J.; Purrazzella, J.; Shields, C.L.; Ganguly, T.; Ganguly, A. Chromosome 3 status combined with BAP1 and EIF1AX mutation profiles are associated with metastasis in uveal melanoma. Investig. Ophthalmol. Vis. Sci. 2014, 55, 5160-5167. [CrossRef]

11. Koopmans, A.E.; Verdijk, R.M.; Brouwer, R.W.; van den Bosch, T.P.; van den Berg, M.M.; Vaarwater, J.; Kockx, C.E.; Paridaens, D.; Naus, N.C.; Nellist, M.; et al. Clinical significance of immunohistochemistry for detection of BAP1 mutations in uveal melanoma. Mod. Pathol. 2014, 27, 1321-1330. [CrossRef] [PubMed]

12. Van Essen, T.H.; van Pelt, S.I.; Versluis, M.; Bronkhorst, I.H.; van Duinen, S.G.; Marinkovic, M.; Kroes, W.G.M.; Ruivenkamp, C.A.; Shukla, S.; de Klein, A.; et al. Prognostic parameters in uveal melanoma and their association with BAP1 expression. Br. J. Ophthalmol. 2014, 98, 1738-1743. [CrossRef]

13. Robertson, A.G.; Shih, J.; Yau, C.; Gibb, E.A.; Oba, J.; Mungall, K.L.; Hess, J.M.; Uzunangelov, V.; Walter, V.; Danilova, L.; et al. Integrative analysis identifies four molecular and clinical subsets in uveal melanoma. Cancer Cell 2017, 32, 204-220. [CrossRef] [PubMed]

14. Dogrusöz, M.; Jager, M.J. Genetic prognostication in uveal melanoma. Acta Ophthalmol. 2017, 96, $331-347$. [CrossRef] [PubMed]

15. Kaliki, S.; Shields, C.; Shields, J. Uveal melanoma: Estimating prognosis. Ind. J. Ophthalmol. 2015, 63, 93-102. [CrossRef] [PubMed]

16. Versluis, M.; de Lange, M.J.; van Pelt, S.I.; Ruivenkamp, C.A.; Kroes, W.G.M.; Cao, J.; Jager, M.J.; Luyten, G.P.M.; van der Velden, P.A. Digital PCR validates 8q dosage as prognostic tool in uveal melanoma. PLoS ONE 2015, 10, e0116371. [CrossRef] [PubMed]

17. Singh, N.; Singh, A.D.; Hide, W. Inferring an evolutionary tree of uveal melanoma from genomic copy number aberrations. Investig. Ophthalmol. Vis. Sci. 2015, 56, 6801-6809. [CrossRef]

18. Decatur, C.L.; Ong, E.; Garg, N.; Anbunathan, H.; Bowcock, A.M.; Field, M.G.; Harbour, J.W. Driver mutations in uveal melanoma associations with gene expression profile and patient outcomes. JAMA Ophthalmol. 2016, 134, 728-734. [CrossRef]

19. Niederkorn, J. Ocular immune privilege and ocular melanoma: Parallel universes or immunological plagiarism? Front. Immunol. 2012, 3, 148. [CrossRef]

20. Maat, W.; Ly, L.V.; Jordanova, E.S.; de Wolff-Rouendaal, D.; Schalij-Delfos, N.E.; Jager, M.J. Monosomy of Chromosome 3 and an inflammatory phenotype occur together in uveal melanoma. Investig. Ophthalmol. Vis. Sci. 2008, 49, 505-510. [CrossRef]

21. Blom, D.J.; Luyten, G.P.M.; Mooy, C.; Kerkvliet, S.; Zwinderman, A.H.; Jager, M.J. Human leukocyte antigen class I expression. Marker of poor prognosis in uveal melanoma. Investig. Ophthalmol. Vis. Sci. 1997, 38, 1865-1872.

22. Ericsson, C.; Seregard, S.; Bartolazzi, A.; Levitskaya, E.; Ferrone, S.; Kiessling, R.; Larsson, O. Association of HLA class I and class II antigen expression and mortality in uveal melanoma. Investig. Ophthalmol. Vis. Sci. 2001, 42, 2153-2156.

23. Jager, M.J.; Hurks, H.M.; Levitskaya, J.; Kiessling, R. HLA expression in uveal melanoma: There is no rule without some exception. Hum. Immunol. 2002, 83, 444-451. [CrossRef]

24. Van Essen, T.H.; van Pelt, S.I.; Bronkhorst, I.H.G.; Versluis, M.; Némati, F.; Laurent, C.; Luyten, G.P.M.; van Hall, T.; van den Elsen, P.J.; van der Velden, P.A.; et al. Upregulation of HLA expression in primary uveal melanoma by infiltrating leukocytes. PLoS ONE 2016, 11, e0164292. [CrossRef] [PubMed]

25. Loumagne, L.; Baudhuin, J.; Favier, B.; Montespan, F.; Carosella, E.D.; Rouas-Freiss, N. In vivo evidence that secretion of HLA-G by immunogenic tumor cells allows their evasion from immunosurveillance. Int. J. Cancer 2014, 135, 2107-2117. [CrossRef] [PubMed]

26. Lesport, E.; Baudhuin, J.; LeMaoult, J.; Sousa, S.; Doliger, C.; Carosella, E.D.; Favier, B. Human melanoma cell secreting human leukocyte antigen-G5 inhibit natural killer cell cytotoxicity by impairing lytic granules polarization toward target cell. Hum. Immunol. 2009, 70, 1000-1005. [CrossRef]

27. Hurks, H.M.H.; Valter, M.M.; Wison, L.; Hilgert, I.; van den Elsen, J.; Jager, M.J. Uveal Melanoma: No expression of HLA-G. Investig. Ophthlalmol. Vis. Sci. 2001, 42, 3081-3084. [PubMed]

28. Gezgin, G.; Dogrusöz, M.; van Essen, T.H.; Kroes, W.G.M.; Luyten, G.P.M.; van der Velden, P.A.; Walter, V.; Verdijk, R.M.; van Hall, T.; van der Burg, S.H.; et al. Genetic Evolution of uveal melanoma guides the development of an inflammatory microenvironment. Cancer Immunol. Immunother. 2017, 66, 904-912. [CrossRef] 
29. Markby, D.W.; Onrust, R.; Bourne, H.R. Separate GTP Binding and GTPase Activating Domains of G $\alpha$ Subunit. Science 1993, 262, 1895-1901. [CrossRef]

30. Bauer, J.; Kiliç, E.; Vaarwater, J.; Bastian, B.C.; Garbe, C.; de Klein, A. Oncogenix GNAQ mutations are not correlated with disease-free survival in uveal melanoma. Br. J. Cancer 2009, 101, 813-815. [CrossRef] [PubMed]

31. Chua, V.; Lapadula, D.; Randolph, C.; Benovic, J.L.; Wedegaertner, P.B.; Aplin, A.E. Dysregulated GPCR signaling and therapeutic options in uveal melanoma. Mol. Cancer Res. 2017, 15, 501-506. [CrossRef] [PubMed]

32. Bronkhorst, I.H.G.; Vu, T.H.K.; Jordanova, E.S.; Luyten, G.P.M.; van der Burg, S.H.; Jager, M.J. Different subsets of tumor-infiltrating lymphocytes correlate with macrophage influx and monosomy 3 in uveal melanoma. Investig. Ophthalmol. Vis. Sci. 2012, 53, 5370-5378. [CrossRef] [PubMed]

33. Amaro, A.; Gangemi, R.; Piaggio, F.; Angelini, G.; Barisione, G.; Ferrini, S.; Pfeffer, U. The biology of uveal melanoma. Cancer Metastasis Rev. 2017, 36, 109-140. [CrossRef] [PubMed]

34. O'Hayre, M.; Degese, M.S.; Gutkind, J.S. Novel insights into G protein and G-protein coupled receptor signalling in cancer. Curr. Opin. Cell Biol. 2014, 27, 126-135. [CrossRef] [PubMed]

35. Wu, X.; Li, J.; Zhu, M.; Fletscher, J.A.; Hodi, F.S. Protein Kinase C inhibitor AEB071 targets ocular melanoma harboring GNAQ mutations via effects on the PKC/Erk1/2 and PKC/NF-KB pathways. Mol. Cancer Ther. 2012, 11, 1905-1914. [CrossRef] [PubMed]

36. Souri, Z.; Wierenga, A.P.A.; van Weeghel, C.; van der Velden, P.A.; Kroes, W.G.M.; Luyten, G.P.M.; van der Burg, S.; Jochemsen, A.G.; Jager, M.J. Loss of BAP1 Is Associated with Upregulation of the NfkB Pathway and Increased HLA Class I Expression in Uveal Melanoma. Cancers 2019, 11, 1102. [CrossRef]

37. Vaqué, J.P.; Dorsam, R.T.; Feng, X.; Iglesias-Bartolome, R.; Forsthoefel, D.J.; Chen, Q.; Debant, A.; Seeger, M.A.; Ksander, B.R.; Teramoto, H.; et al. Genome-wide RNAi screen reveals a Trio-regulated Rho GTPase circuitry transducing mitogenic signals initiated by G protein-coupled receptors. Mol. Cell 2013, 49, 94-108. [CrossRef]

(C) 2019 by the authors. Licensee MDPI, Basel, Switzerland. This article is an open access article distributed under the terms and conditions of the Creative Commons Attribution (CC BY) license (http://creativecommons.org/licenses/by/4.0/). 